\title{
L'œil classique, sous la direction de Sylvaine Guyot et Tom Conley
}

\section{Monica Pavesio}

\section{Q OpenEdition}

1 Journals

\section{Edizione digitale}

URL: http://journals.openedition.org/studifrancesi/4357

DOI: 10.4000/studifrancesi.4357

ISSN: 2421-5856

\section{Editore}

Rosenberg \& Sellier

\section{Edizione cartacea}

Data di pubblicazione: 1 settembre 2016

Paginazione: 323-325

ISSN: 0039-2944

\section{Notizia bibliografica digitale}

Monica Pavesio, «L'œil classique, sous la direction de Sylvaine Guyot et Tom Conley », Studi Francesi

[Online], 179 (LX | II) | 2016, online dal 01 septembre 2016, consultato il 18 septembre 2020. URL :

http://journals.openedition.org/studifrancesi/4357 ; DOI : https://doi.org/10.4000/studifrancesi.4357

Questo documento è stato generato automaticamente il 18 settembre 2020.

\section{(c) (i) (9)}

Studi Francesi è distribuita con Licenza Creative Commons Attribuzione - Non commerciale - Non opere derivate 4.0 Internazionale. 


\title{
L'œil classique, sous la direction de Sylvaine Guyot et Tom Conley
}

\author{
Monica Pavesio
}

\section{NOTIZIA}

L'œil classique, sous la direction de Sylvaine GUYOT et Tom CONLEY, «Littératures classiques» 82, 2013, $314 \mathrm{pp}$.

1 Il numero monografico di «Littératures classiques» riunisce diciotto contributi dedicati, come annuncia Sylvaine Guyot nella prefazione, all'“Ancien règime du voir", ossia ai meccanismi di sviluppo, di fabbricazione e di divulgazione dello sguardo nella Francia detta "classica", al modo di pensare e di rappresentare la visione, considerata dal punto di vista della percezione e della configurazione del visibile. Si tratta, insomma, di rileggere il xviI secolo francese nell'ottica dei visual studies, di analizzare come la riflessione sulla visione sia accompagnata, nel Seicento, da una serie di contraddizioni interne che gli studi riuniti hanno lo scopo di evidenziare. L'aggettivo "classico" è utilizzato dai curatori come semplice indicazione cronologica e non estetica, anche se, come ben sottolinea Viala nel primo studio della raccolta, la delimitazione del periodo denominato "âge classique" (tutto il XVII secolo?, solo il XVII secolo?), risulta, oggi più che mai, discutibile.

2 I diciotto contributi avrebbero potuto essere organizzati in funzione dell'oggetto sul quale lo sguardo si posa: il teatro (saggi di Biet, Bilis, Cherbuliez, Guyot, Thouret); l'iconografia (studi di Ravel, Roussillon, Viala); i fenomeni naturali (articoli di Goldstein, Lyons); la visione dell'alterità sociale, etica e sessuale (saggi di Bertrand, Greenberg, Longino, Reguig); la visione della storia (studio di Régent-Susini); la visione del testo (articoli di Blanchard, Conley, Peters). Si è preferito, invece, sistemarli seguendo un percorso che tiene conto delle quattro problematiche ritenute fondamentali per illustrare le caratteristiche dell'“Ancien régime du voir": «Aux bords du siècle», sezione che esplora come l'identità e la coscienza storica di un'epoca appaia 
nelle immagini che tale epoca produce; «Multiplicités», dedicata ad una caratteristica spesso trascurata dell'epoca classica: la mobilità, la frammentazione e l'eterogeneità dei punti di vista; «Frontière», gruppo di studi incentrato sui limiti (empirici, teorici, normativi), che impone una visione comune; «Actes de régard», capitolo conclusivo dedicato all'analisi dei rapporti tra lo sguardo e l'ordine imposto dal potere.

La prima sezione, "Aux bords du siècle», si apre con un interessante studio retrospettivo di Alain VIALA (Inventer Watteau) che, partendo dall'analisi di tre quadri di Watteau dipinti intorno al 1715, reperisce la coesistenza nel Seicento di due "savoirvoir": il modello maschile e accademico che impone alle immagini la coerenza di un ordine stabilito, e l'enigma, femminile e galante, che resiste alle imposizioni e spinge lo spettatore a creare la propria visione. Il saggio successivo di Tom CONLEY (L'oeil épluche. Voir chez Béroalde de Verville) analizza un testo, Le Palais du curieux di Béroalde de Verville, scritto all'inizio del secolo, che presenta un curioso percorso in cui il libro si trasforma in un quadro, nel quale si trovano riuniti fenomeni differenti che possono essere considerati e interpretati liberamente. L'ultimo articolo di Jeffrey S. RAVEL (Trois images de l'expulsion des Comédiens italiens en 1697), ritorna sugli anni cerniera della fine del XVII secolo e l'inizio del XVIII, per esaminare, grazie a tre immagini anonime e non datate, come l'espulsione dei commedianti italiani da Parigi, attuata da Luigi XIV nel 1697 , sia stata riletta e interpretata negli anni successivi.

4 Cinque studi afferiscono alla seconda sezione della miscellanea, dedicata all'eterogeneità dei punti di vista e alle contraddizioni presenti nell'epoca classica. Lo studio di Mitchell GREENBERG dal suggestivo titolo Classicisme hard. Vision et différence sexuelle au XVII siècle, mette in luce come il modo di concepire la sessualità cambi profondamente con l'avanzare del secolo, partendo da una visione aperta senza distinzione fissa tra i due sessi, per arrivare a una concezione eterosessuale più rigida del desiderio umano, non senza ambivalenze interne che l'assolutismo cercherà di mitigare.

5 I due articoli successivi sono dedicati al teatro. Nel primo, Christian BIET (Séance, performance, assemblée et représentation: les jeux de regards au théâtre, XVII ${ }^{e}-\mathrm{XXI}{ }^{e}$ siècle), studia come il "mouvement oscillatoire" degli sguardi a teatro presenti una tensione che nasce dalla realizzazione delle due funzioni tradizionali dell'arte: quella unificatrice di un legame sociale armonioso e ortodosso e quella eterogenea e critica di un luogo, in cui questo tentativo di ordine è messo in discussione. Clotilde THOURET, nel secondo studio (Voir, juger, découvrir: la place du regard dans la Querelle du Cid), dipinge lo sguardo dello spettatore come nodo polemico della Querelle del Cid, nella quale si oppongono l'idea antica di un legame stretto tra la percezione passiva dello spettatore e la visione teorica dei dotti, e la rivendicazione moderna di un nuovo legame tra vista, effetto prodotto dalla rappresentazione teatrale e giudizio espresso senza condizionamenti.

Dominique BERTRAND, con il suo saggio (Le risible et le visible dans l'"Histoire comique de Francion": évidence ou discordance?) analizza le strategie visive utilizzate da Sorel per provocare il riso dei lettori, riflettendo sull'ambivalenza che permane da parte dell'autore nell'affrontare le tematiche erotiche e oniriche.

7 Nell'ultimo studio di questa sezione, Sylvaine GUYOT (Entre éblouissement et 'véritables grâces'. Racine ou les tensions de l'œil classique) ritorna al teatro, per affrontare il tema del sublime che provoca l'adesione incondizionata degli spettatori del Grand Siècle, definito, 
a partire da Voltaire, l'identità visiva del classicismo. La studiosa trova, in alcune pièces di Racine, delle "scénographies visuelles critiques" in cui il drammaturgo esplora il paradigma dello stupore nei suoi limiti e nei suoi eccessi, facendo emergere una visione toccante e magnetica, generatrice di desiderio e piacere dei sensi, che supera il supposto predominio del sublime, per attirare gli sguardi degli spettatori, a volte emozionati, a volte sconcertati, ma mai passivi.

8 La terza sezione, denominata «Frontières», si apre con uno studio di John D. LYoNS sulle frontiere del visibile in Pascal (Pascal et les frontières du visible). In un'epoca in cui le scoperte scientifiche permettono di migliorare la visibilità dei fenomeni celesti, si apre un forte dibattito epistemologico e teologico tra visibile e invisibile che Lyons ritrova e analizza in alcuni frammenti delle Pensées. Il secondo articolo di Claire GOLDSTEIN (Le regard en déroute: la comète de 1680), riprende l'argomento precedente, indagando come, in una società eliocentrica, con il Re Sole al centro dell'Universo, le scoperte scientifiche generino angosce inedite, e la cometa apparsa nel 1680 provochi, negli scritti dell'epoca, un'inquietudine legata alla nuova possibilità di osservare ciò che fino ad allora era sconosciuto.

Portando la riflessione su un'altra frontiera, Juliette CHERBULIEZ («Et que méconnaîtrait l'œil même de son père»: les limites du savoir oculaire dans la description tragique) analizza i récits tragiques del teatro classico, reperendo in alcuni di essi più che l'utilizzo di codici di una retorica visiva, la predominanza di una rappresentazione che interpella il senso della tattilità, più efficace a livello patetico.

In un registro ancora diverso, Jeffrey PETERS (Orphée en devenir: image et invention dans les "Fables" de La Fontaine) legge le Fables di La Fontaine, servendosi del mito di Orfeo, per illustrare come in esse l'invenzione sia collegata alla problematica dello scarto, grazie al quale l'immagine partecipa a un movimento che fa della favola un gioco di distanze e di angolazioni ottiche sempre incomplete e mai ultimate.

11 Nell'ultimo articolo della sezione (Perspectives dépravées, perspectives rectifiées: l'histoire universelle peinte par Bossuet), Anne RÉGENT-SUSINI studia la pedagogia fortemente visiva presente nei Discours sur l'histoire universelle scritti da Bossuet per il Delfino, per aiutare il Principe ad avvicinarsi all'unico e solo punto di vista che gli permetterà di comprendere come gli avvenimenti formino sempre un quadro coerente.

12 L'ultima sezione si apre con un articolo di Jean-Vincent BLANCHARD (De quoi donner une jaunisse à Richelieu. Autour d'une lettre de Descartes à Guez de Balzac), in cui si sottolineano gli effetti politici e filosofici della metafora ottica in una lettera di Descartes a Balzac del 1631, sull'autonomia del singolo nei confronti dello stato.

13 I due saggi successivi sono dedicati alle procedure di visualizzazione dei valori politici. Hélène BILIs (Voir la Sorcière de Colchis d'un nouvel ceil, ou comment rendre la dignité visible), studiando Médée, osserva come Corneille si sforzi, all'inizio della sua carriera, di rendere manifesta la dignità singolare della figura reale. Marine RoussiLlon (Que voit-on dans les poèmes héroïques des années 1650?), grazie allo studio dei paratesti e delle incisioni di quattro poemi eroici di metà Seicento, conclude che l'attualità dei valori monarchici assunti da eroi antichi emerge in scene di visioni che mettono in relazione il presente con il passato.

14 Gli ultimi due testi s'interrogano sulla labilità dei rapporti di forza orchestrati dalla teatralità socio-politica. Michèle LoNGino (Le voyageur, les eunuques et le sérail: l'oculaire par procuration) studia la Nouvelle Relation de l'interieur du Serrail du Grand Seigneur (1675), 
scritta da Tavernier, un ricco mercante e grande viaggiatore, per arrivare alla conclusione che la testimonianza oculare di due testimoni viene filtrata da Tavernier, e la descrizione del serraglio appare come uno specchio paradossale della corte di Luigi XIV. Delphine REGUIG (L'oeil du maître: regarder les "Fables" de La Fontaine) dimostra come nelle Fables di La Fontaine la messa in scena degli sguardi sia sempre collegata a un processo di svelamento, in cui il lettore assiste, per così dire, alle Fables, perché, come a teatro, le guarda e le interpreta. 